\title{
Non-Hodgkin's Lymphoma in Thyroid Gland
}

\author{
MD.MAHABUBUR ROSHED, ${ }^{1}$ SK. MANOWAR AHSAN, ${ }^{2} \mathrm{KAZI} \mathrm{ABU} \mathrm{RASHED}^{3}$
}

\begin{abstract}
A rare case of primary non-Hodgkin's Lymphoma in a 52-year-old female presented with a large mass in the right lobe of thyroid. The diagnosis was established by fine needle aspiration cytology (FNAC) followed by surgery and finally confirmed by histopathology.
\end{abstract}

Keywords: Lymphoma, FNAC, Thyroid scan, Ultrasonography (USG).

\section{Introduction}

The non-Hodgkin's lymphomas are extremely rare thyroid neoplasms. Of all thyroid malignancies $1 \%$ to $3.5 \%$ are malignant lymphomas ${ }^{1}$. The diagnosis of a primary thyroid non-Hodgkin's lymphomas is made only by exclusion, after a thorough clinical examination and radiological investigations. The tumor needs to be differentiated from other poorly differentiated neoplasms that share a similar morphological picture ${ }^{2}$, at present, about 54 cases of primary non-Hodgkin's lymphomas of the thyroid, both in adult and children have been documented in the literature ${ }^{3,8}$. This report describes a case of a primary thyroid non-Hodgkin's lymphomas presenting as a large thyroid mass that was diagnosed by FNAC.

\section{Case Report}

A 52-year-old house wife presented with a right-sided painless thyroid swelling for the last 2 months. On general physical examination there was firm to hard irregular mass in the right lobe of thyroid occupying lateral margin to lower pole. Other systemic examination was unremarkable. Investigations revealed a normal haemogram, chest skiagram, blood biochemistry and a nonspecific electrocardiogram (ECG). Ultrasonography of thyroid showed a complex mass consisting of hypo and hyper echoic areas measuring $6.0 \times 3.9 \mathrm{~cm}$, occupying lateral and lower parenchyma of the right lobe of thyroid suggesting a cystic degeneration and areas of calcification. The left lobe of thyroid appeared normal and there was no focal lesion or lymphadenopathy. However, hormonal assay reveal subclinical hypothyroidism due to increased THS level with

1. Lecturer, Department of Pathology, Khulna Medical College, Khulna.

2. Assistant Professor, Department of Pathology, Khulna Medical College, Khulna.

3. Junior consultant, Department of ENT, General Hospital, Khulna.

Correspondence : Dr. Md.Mahabubur Roshed, Lecturer, Department of Pathology, Khulna Medical College, Khulna, Email: roshedm@yahoo.com normal $\mathrm{T}_{3}$ and $\mathrm{T}_{4}$ level. Thyroid scan with $\mathrm{I}^{125}$ show low radiotracer uptake in right lobe of thyroid. Most of the cases, the patients are euthyroid and the tumor present as one or more cold nodule on thyroid scan, a very high proportion of primary thyroid lymphomas arise in the background of lymphocytic or Hashimoto's thyroiditis. ${ }^{3}$ Primary malignant lymphoma of thyroid usually arises in an immunologically abnormal gland, usually affected by chronic lymphocytic thyroiditis. ${ }^{1}$ Ultrasound of the abdomen revealed no abnormality.

Fine needle aspiration cytology of the mass reveals highly cellular material composed of atypical monotonous lymphocytes arranged in diffusely that had large hyper chromatic nuclei, granular chromatin and irregular nuclear membrane, against a hemorrhagic and necrotic background, cells in mitosis are also seen.(Figure-1). Histological examination of tissue sections revealed loss of nodal architecture due to diffuse infiltration of atypical large lymphoid cell. There was cytological evidence of malignancy and up to 15 mitoses per 10 high-power fields were seen in most cellular areas.(Figure-2). These features were compatible with non-Hodgkin's lymphoma. ${ }^{3}$

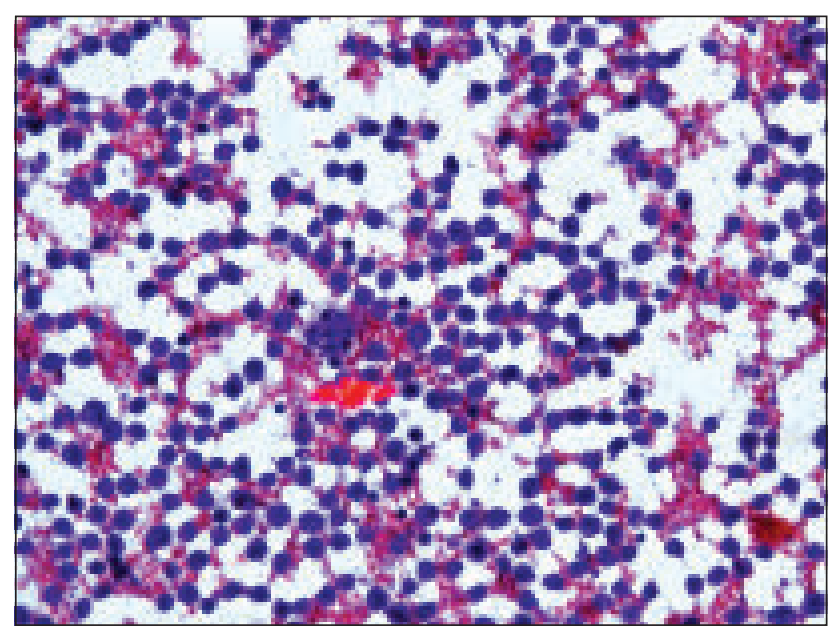

Fig.-1: Photomicrograph of FNA showing a case of NonHodgkin's lymphoma. 


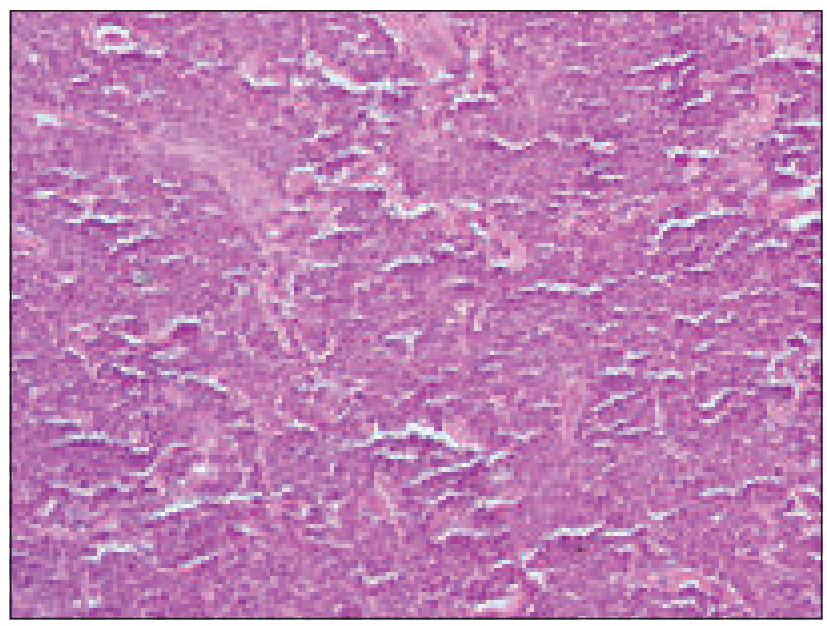

Fig.-2. Photomicrograph of biopsy showing atypical monotonous lymphoid cells arranged diffusely and showing cytological evidence of malignancy (Haematoxylin-Eosin x 100)

\section{Discussion}

Non-Hodgkin's lymphoma of the thyroid is an extremely rare neoplasm that can arise from the thyroid parenchyma. Most extra nodal non-Hodgkin's lymphomas are seen in the sot tissue in elderly patient and rare in glandular organ. ${ }^{4}$ Most cases of primary non-Hodgkin's lymphoma of thyroid are seen in adult or elderly female; thyroid enlargement is often rapid and can lead to symptom of tracheal or laryngeal compression $^{3}$. Clinically, thyroid lymphoma affects women more frequently than men (ratio 2.5 to $8.4: 1$ ). ${ }^{1}$ Intrathyroid Non-Hodgkin's lymphomas often do not cause any symptoms, especially when small. Radiologically these present as a smooth or lobulated hypoechoic mass distinguishable from thyroid parenchyma. The nonHodgkin's lymphoma usually presents with irregular firm to hard thyroid swelling with or without pain, and radiologically may show complex mass consisting of hypoechoic and hyper echoic areas..$^{5}$ The presentation of the present case is usual, as the patient had a large irregular hard thyroid mass, almost completely occupying the lateral and lower pole of right lobe of thyroid, with a characteristic ultrasonographic appearance of a circumscribed hypoechoic and hyperechoic complex mass with calcified areas. The cytological features of non-Hodgkin's lymphoma of thyroid includes atypical monotonous lymphoid cells with granular chromatin and irregular nuclear membrane. ${ }^{6}$ The differential diagnosis of non-Hodgkin's lymphoma of thyroid include lymphocytic thyroiditis and other carcinomas, such as medullary carcinoma, follicular carcinoma and poorly differentiated carcinoma. ${ }^{7}$ However, tumors other than non-Hodgkin's lymphoma exhibit specific cellular arrangement, individual cellular characteristics, such as follicular carcinoma show follicular arrangement and mild to moderate pleomorphism, medullary carcinoma show minimum affinity of cellular clustering and spindle shape or plasmacytoid appearance of individual cell, poorly differentiated carcinoma reveals cellular clustering, scanty cytoplasm and conspicuous nucleoli, papillary carcinoma show papillary arrangement, nuclear groove and pseudo nuclear inclusion. ${ }^{3}$

The prognosis of these tumors is related to the tumor size, tumor grade, mitotic count and age of the patient. Tumors occur in children and young adults have a better prognosis. ${ }^{8}$

High grade tumor often behaves in a highly malignant fashion with death occurring within 2.5 years. Tumors with ${ }^{3} 8$ mitotic figures per 10 high-power fields behave in an aggressive fashion while others remain stationary or tend to grow slowly. ${ }^{9}$ Non-Hodgkin's lymphoma have high incidence into the distant lymphnode, although these have tendency for local extension into the adjacent soft tissue. By virtue of their rapid extension, death occurs within two years. ${ }^{6.9}$ Guccion and Rosen analyzed 13 cases of intra thyroid non-Hodgkin's lymphoma. The largest high grade tumor was $13 \mathrm{~cm}$ in size and the patient died in four months in contrast to cases with low grade tumors of $3.5 \mathrm{~cm}$ size where the patients survived for more than two years. On the other hand, Fischer et al reported a 61-year-old male with a 'giant high grade non-Hodgkin's lymphoma' involving the whole thyroid and the surrounding soft tissue where the patient underwent three extended resections including the skeletal muscle followed by radiotherapy and chemotherapy after last resection. This patient survived with no signs of recurrence after five years of the last resection, favoring multiple extended resections for large and aggressive high nonHodgkin's lymphoma.

\section{Conflict of Interest : None}

\section{References}

1. Young R.H., Clement P.B, Scully R.E., 1999. 'Diagnostic Surgical Pathology. $3^{\text {rd }}$ edition. Sternberg S.S., Antonioli D.A.,Carter D., Mills S.E and Oberman H.A(eds). Lippincott Willams and Willkins, Philadelphia.

2. Marcus, et al. Lymphoma - Pathology, Diagnosis and Treatment. 2007. Cambridge University Press.

3. Rosai J.2004. Ackerman's Surgical Pathology. $9^{\text {th }}$ edition. CV. Mosby Company.

4. Full Guidance On Rituximab For Aggressive Non-Hodgkin Lymphoma. September 2003. National Institute for Health and Clinical Excellence (NICE).

5. DeVita, Vincent T. et al. (2008) Cancer: principles and practice of oncology, 8th edition. Philadelphia: Wolters Kluwer/ Lippincott Williams \& Wilkins.

6. Canellos, George et al. (editors) (2006) The Lymphomas, 2nd edition. Philadelphia: Saunders/Elsevier.

7. Poston, Graeme et al. (editors) (2007) Textbook of Surgical Oncology. London: Informa Healthcare.

8. Raghavan, Derek et al. (editors) (2006) Textbook of Uncommon Cancer, 3rd edition. Hoboken, NJ: John Wiley \& Sons.

9. Myers, Eugene et al. (editors) (2003) Cancer of the Head and Neck, 4th edition. Philadelphia: Saunders. 